$\begin{array}{cl}\begin{array}{c}\text { Revue } \\ \text { de /histoire }\end{array} & \text { Revue de l'histoire des religions } \\ \text { des religions } & \begin{array}{l}3 \mid 2011 \\ \text { Varia }\end{array}\end{array}$

Délos-Thasos : un aller-retour paradoxal dans le matériel votif

Delos-Thasos : a Paradoxical Round-Trip in Votive Material

\title{
Clarisse Prêtre
}

\section{OpenEdition}

1 Journals

Édition électronique

URL : http://journals.openedition.org/rhr/7783

DOI : $10.4000 /$ rhr.7783

ISSN : 2105-2573

Éditeur

Armand Colin

Édition imprimée

Date de publication : 1 septembre 2011

Pagination : 331-348

ISBN : 978-2200-92721-9

ISSN : 0035-1423

Référence électronique

Clarisse Prêtre, «Délos-Thasos : un aller-retour paradoxal dans le matériel votif », Revue de l'histoire des religions [En ligne], 3 | 2011, mis en ligne le 01 septembre 2014, consulté le 19 avril 2019. URL : http:// journals.openedition.org/rhr/7783; DOI : 10.4000/rhr.7783 


\section{Délos-Thasos : un aller-retour paradoxal dans le matériel votif}

Si les liens historiques entre les Cyclades et l'île de Thasos se marquent par des biais évidents (céramique, sculpture), il est un domaine de comparaison qu'on oublie trop souvent, celui du matériel votif. Les offrandes dans les sanctuaires thasiens présentent des similitudes intéressantes avec les realia décrits dans les inventaires des sanctuaires déliens. En partant de quelques termes recensés dans les inscriptions, on tentera de trouver leurs parallèles dans les nombreuses offrandes livrées par les fouilles de l'Artémision thasien et on verra aussi si certains objets thasiens comportent un correspondant délien. Ce sera donc l'occasion de souligner l'intérêt des études qui allient épigraphie et archéologie.

\section{Delos-Thasos : a Paradoxical Round-Trip in Votive Material}

While the historical links between the Cyclades and the isle of Thasos are seen in obvious fields (ceramic, sculpture), there is a field of comparison too often forgotten, votive material. Offerings in Thasian sanctuaries have interesting similarities with the realia described in the inventories of Delian sanctuaries for example. On the basis of some terms noted from engravings, I will attempt to find their parallels in the numerous offerings found in the excavations of the Thasian Artemision, and equally to see if certain Thasian offerings have a Delian parallel. This will thus provide an occasion to emphasize the interest of studies that combine epigraphy and archaeology. 
Depuis de nombreuses décennies, les fouilles menées par le service archéologique grec et par l'École française d'Athènes contribuent à mieux comprendre l'histoire religieuse de Thasos: des inscriptions sur les rites cultuels sont découvertes régulièrement, les sanctuaires principaux sont connus, et le matériel céramique et plastique est étudié avec minutie.

Mais en matière de religion, seules ont été menées jusqu'à présent des études synthétiques ${ }^{1}$ ou au contraire très exclusives sur un sanctuaire, voire sur une seule partie de son matériel. Or, dans ce matériel, il est un domaine très peu exploité mais qui constitue pourtant l'un des fondements du système votif. C'est ce qu'on nomme souvent péjorativement «les petits objets», c'està-dire, en donnant une définition par la négative, tout ce que les spécialistes de céramique, de numismatique, de terre cuite ou de sculpture ne peuvent prendre en compte dans leurs études: selon les sites, cela va des bijoux aux outils, avec comme point commun leur petite taille et surtout, le fait qu'ils ont été trouvés en contexte votif ${ }^{2}$. Cet article est aussi l'occasion de combattre vigoureusement l'emploi de l'expression "petits objets », très dépréciative et qui ne reflète pas la richesse et la variété de ce domaine. On préfèrera l'expression «matériel votif», voire «instrumentum votif» dans certains cas.

L'étude des realia trouvés au cours des fouilles ne saurait toutefois suffire à la compréhension du phénomène dédicatoire parce qu'il est difficile de toujours percevoir le motif du don derrière l'objet. Les inventaires épigraphiques constituent l'heureux pendant de la démarche archéologique en renseignant non seulement sur le matériel dédié mais aussi sur le contexte votif, sur le donateur et sur la divinité bénéficiaire ${ }^{3}$.

1. Notamment Jean Pouilloux, Recherches sur l'histoire et les cultes de Thasos. I. De la fondation de la cité à 196 av. J.-C. Paris, 1954; Jean Pouilloux, Christine Dunant, Recherches sur l'histoire et les cultes de Thasos. II: de 196 avant J.-C. jusqu'à la fin de l'Antiquité, 1958.

2. Donc dans les sanctuaires le plus souvent, mais nous n'excluons pas le matériel des tombes comme fonds de comparaisons.

3. Nous faisons allusion ici à l'importance des épiclèses mentionnées dans les inventaires et qui permettent parfois de comprendre la raison de certaines dédicaces à des divinités en apparence peu concernées. 
Il s'agira donc ici de jeter une passerelle entre les mentions d'offrandes dans les sources épigraphiques et leurs correspondants trouvés au cours des fouilles, c'est-à-dire dans le cas présent, dans les fouilles de l'Artémision thasien ${ }^{4}$. Au-delà de résultats aléatoires, comme l'illustrera le second exemple de cette étude, le but est de démontrer que pour être mieux compris, toute pratique votive et tout objet votif doivent être remis en perspective dans un cadre plus large que celui du sanctuaire auquel ils appartiennent et qu'on ne saurait se cantonner à une analyse exclusive, qu'elle se fonde sur l'archéologie ou l'épigraphie.

Pour appréhender l'instrumentum votif de l'Artémision, il est nécessaire de dépasser les limites de l'île et de se projeter dans le contexte cultuel de Paros, logiquement, mais aussi dans un modèle de pratiques dédicatoires bien cerné. Les inventaires déliens constituent le substrat idéal pour cette étude : chaque année, des dédicants venus du monde grec - et de bien au-delà aussi - déposaient leurs dons aux multiples divinités honorées sur l'île. Les donateurs les plus nombreux étaient, outre les Déliens, ceux qui venaient des îles des Cyclades les plus proches, soit Naxos et Paros. Depuis longtemps on connaît la présence d'un Delion à Paros ${ }^{5}$, et une inscription du $\mathrm{IV}^{\mathrm{e}} \mathrm{s}$. récemment mise au jour à Thasos révèle également l'existence d'un Delion thasien ${ }^{6}$. On voit alors clairement se dégager le lien qui permet de relier les objets votifs des fouilles thasiennes aux realia des inventaires déliens. Paros en est peut-être le dénominateur commun, mais même au-delà de son rôle de métropole, on sait à présent qu'il existait de réelles connexions votives entre les trois îles.

En partant de ce constat, il semblait intéressant de relever dans les inscriptions déliennes les mentions des donateurs pariens et éventuellement thasiens.

Sur l'ensemble du corpus des comptes et inventaires déliens, trente-cinq personnes sont décrites avec l'ethnique Пó@ıs. Parmi

4. Nous avons la responsabilité de la publication du matériel votif de ce sanctuaire thasien dans le cadre de l'Ecole française d'Athènes.

5. Otto Rubensohn, Das Delion von Paros (1952); Manfred Schuller, Der Artemistempel im Delion auf Paros (1991).

6. François Salviat, Yves Grandjean «Règlements du Délion de Thasos», Bulletin de Correspondance Hellénique 130 (2006), p. 293-328. 
elles, se trouvent des entrepreneurs, des architectes, des comédiens, des personnes à la qualité indéterminée (seulement «père de», ou «fille de») et près d'un tiers des Pariens nommés représente un donateur, un individu, venu prier ou apporter son offrande sur l'île sacrée ${ }^{7}$. Sur le nombre, il n'y a que deux femmes et il faut insister sur ce point pour souligner encore et toujours que le type d'offrande n'a pas forcément de rapport avec le sexe du donateur.

En revanche, les potentiels donateurs thasiens ne sont jamais mentionnés expressément dans les inventaires et tout au plus

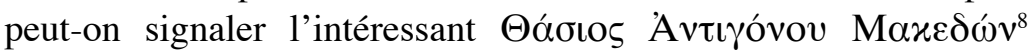
inconnu bien sûr dans la prosopographie thasienne mais qui présente une nomenclature évoquant directement Antigonos Gonatas de Macédoine, fils de Démétrios Poliorcète; Antigonos Gonatas a d'ailleurs dédié de nombreuses offrandes à Délos dans les années 260-250, soit à peu près à la même époque que le personnage

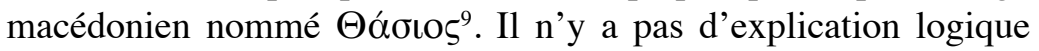
à l'absence de dédicants thasiens dans les inventaires déliens: rappelons simplement que les inventaires n'étaient pas reproduits de façon exhaustive chaque année, et que d'autre part, les hiéropes et autres administrateurs ne mentionnaient pas systématiquement les ethniques des donateurs.

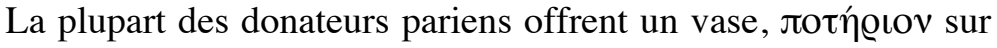
lequel se trouve gravé leur nom; c'est l'offrande la plus commune avec la phiale. Un seul, Médôn, dédie un statère de Sicyone,

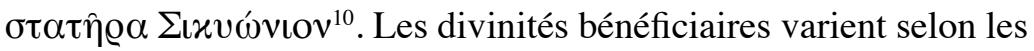
époques et outre Apollon et Artémis sous l'Indépendance, ce sont les divinités égyptiennes, Sarapis, Isis et Anoubis, qui reçoivent les dons des Pariens sous la seconde domination athénienne.

Le passage en revue de ces dédicants pariens reconnus à Délos permet surtout de constater qu'ils n'ont aucune particularité votive en rapport avec leur provenance. Leurs dons sont identiques à l'ensemble enregistré dans les inventaires, il n'y a pas de formule de consécration marquée, pas de rites particuliers ni de donateurs

7. Inscriptions de Délos 53 (3); ID 54; ID 103 (12);ID 298 A (58); Inscriptiones Graceae XI 4, 1221; ID 1403 Bb, II (60); ID 1442 A (71).

8. ID $1403 \mathrm{Bb}, \mathrm{II}(65)$.

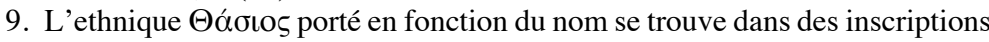
attiques d'époque impériale, par exemple ( $\left.I G \mathrm{II}^{2} 3,2,9995\right)$.

10. ID $103(12)$ 
célèbres. Force est de reconnaître qu'ils n'ont d'ailleurs aucune raison de se distinguer des autres. Cela autorise donc à élargir le champ de comparaison entre les offrandes déliennes et les objets thasiens à l'ensemble des termes de realia relevés dans les inscriptions de Délos, même si on regarde avec plus d'attention, toutefois, les offrandes dédiées à Artémis.

Il est bien sûr hors de question d'analyser ici tous les termes des inventaires déliens qui pourraient avoir leurs correspondants archéologiques à Thasos et nous retiendrons deux offrandes, dont l'étude présente des similitudes intéressantes: l'une, appartenant au domaine de l'instrumentum, est souvent citée à Délos avec des variantes formelles pour l'objet, mais offre des parallèles thasiens assez peu diversifiés ; l'autre, relevant du domaine de la parure, est une offrande bien répertoriée à Délos, mais les rapprochements avec des objets thasiens sont encore plus malaisés à établir. Ce sera donc l'occasion ici de souligner une fois encore l'importance de l'interaction entre ces deux disciplines complémentaires que sont l'archéologie et l'épigraphie.

Les donateurs qui venaient à Délos n'offraient pas seulement des objets à vocation purement dédicatoire, ni même d'objets à caractère ostentatoire: l'acte de «piété » se marquait aussi bien par des offrandes déjà utilisées que par des objets brisés auxquels il fallait alors accorder une valeur affective évidente. Aussi il n'était pas anormal d'enregistrer dans les catalogues un crochet à viande, une quenouille ou encore un ciseau ${ }^{11}$. C'est ainsi qu'on relève également le don de diverses clefs, scrupuleusement enregistrées dans les inventaires. On peut s'interroger sur la réalité de la fonction de cet objet d'autant que certains exemplaires appartiennent de manière évidente aux portes d'un sanctuaire et que les comptes mentionnent la fabrication de clefs ${ }^{12}$. Mais la facture soignée de

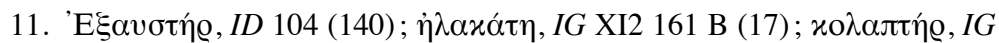
XI2 223 B (31).

12. ID 144 B (17) par exemple. Pour une liste complète, voir MarieChristine Hellmann, Recherches sur le Vocabulaire de l'Architecture d'après

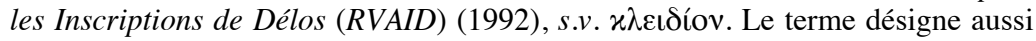
bien le verrou que la clef simple. Selon la définition de Hellmann, une clef est nécessaire quand on a besoin de fermer un édifice de l'extérieur, comme dans le cas d'un temple par exemple. Quand la kleis est dite placée sur un bâtiment, il s'agit plus sûrement d'un verrou. Nous avons donc opté pour la traduction par 
chacun, la description qui en est faite et surtout, la place dans l'inventaire, au sein de l'énumération d'autres offrandes, tout cela dénote un acte votif probable: l'utilisation d'une offrande n'est en rien incompatible avec l'intention première, si cela concerne le dieu et son sanctuaire. Qu'on songe simplement aux vêtements dédiés pour orner les statues de culte et on pourra appliquer ce mode de fonctionnement à d'autres dédicaces. Gardons-nous de tout anachronisme dangereux qui tendrait à considérer qu'une offrande revêt un caractère intouchable: la preuve en est aussi la refonte d'anciens dons ou l'échange d'autres qui «désacralisent» à raison l'objet dédié.

On relève différentes clefs enregistrées dans les inventaires déliens ${ }^{13}$ :

- dans l'entrée du sanctuaire d'Agathè Tychè, sur les portes

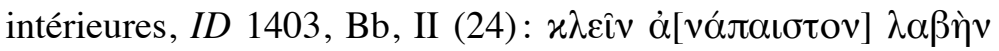

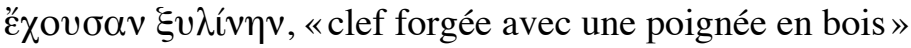

- dans le Kynthion, à la suite de la mention du portail d'entrée de

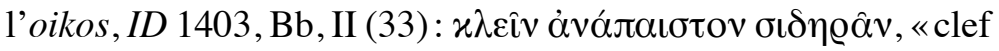
forgée en fer»

- dans l'Aphrodision de Stésiléos, à la suite d'un heurtoir, ID 1414,

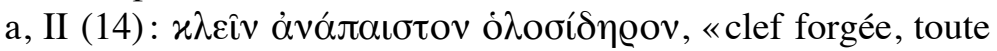
en fer ${ }^{14}$

- dans le sanctuaire d'Anios, avant une statuette d'Apollon, $I D$

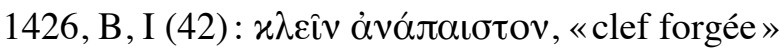

- dans l'Aphrodision de Stésiléos, à la suite de trois paires de

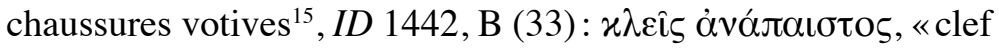
forgée»

- dans l'Artémision, à la suite d'un vêtement pour la statue de

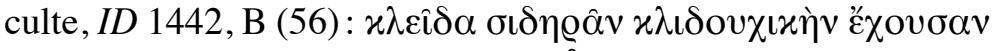

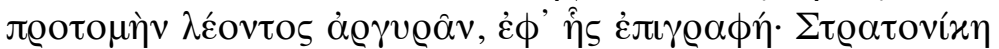

«clef» dans les cas déliens que nous traitons ici, sans que cela puisse s'appliquer à toutes les autres occurrences.

13. Les occurrences intermédiaires des offrandes ne sont pas mentionnées. Seules sont citées les premières mentions d'un nouveau don.

14. Plutôt que «clef entièrement en fer forgé», M.-Chr. Hellmann, op. cit. et à sa suite, Cécile Durvye, "Aphrodite à Délos, Culte privé et public à l'époque hellénistique», Revue des Etudes Grecques 119 (2006), p. 103; l'expression n'insiste pas sur la facture de la clef mais bien sûr son matériau, en opposition aux clefs avec une poignée en bois.

15. i.e. à fonction uniquement dédicatoire, et non des chaussures usuelles. 


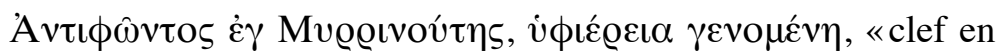
fer de cleidouque, avec une protomée de lion en argent, portant l'inscription: Stratonikè fille d'Antiphôn, de Myrrhinonte, devenue sous-prêtresse».

Il est notable que seuls les inventaires de la seconde domination athénienne mentionnent ces clefs parmi les offrandes. Il ne faut pas pour autant en déduire un argument $a$ silentio: cela témoigne juste d'un changement dans les mentalités qui s'est répercuté dans les recensements enregistrés. Après la rigueur des hiéropes se cantonnant strictement aux dédicaces «mobiles» déposées sur les étagères des temples, les administrateurs athéniens ont ajouté dans les listes tous les éléments fixes qui avaient pu être dédiés longtemps auparavant pour servir d'offrande «utile» et qui faisaient désormais partie intégrante des sanctuaires ${ }^{16}$.

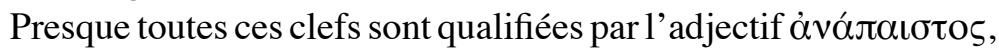
dérivé du verbe $\pi \alpha i ́ \omega$ «frapper, battre» qui désigne, à Délos uniquement, une technique de martelage du métal qui existait sans doute ailleurs. Une fois encore ${ }^{17}$ néanmoins, les rédacteurs des inventaires ont fait preuve d'originalité en utilisant un terme qui n'est attesté dans les sources littéraires que pour désigner un type de pied métrique, l'anapeste.

Le modèle de clef est rarement décrit avec précision: une seule fois, on signale qu'un exemplaire a un «manche» en bois. Le terme $\lambda \alpha \beta$ í s'applique à de nombreuses offrandes dans les inventaires déliens et leurs correspondants attiques, des chasse-

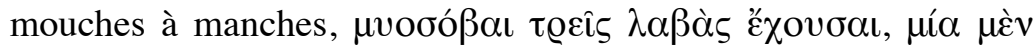

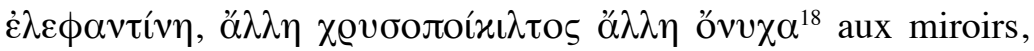

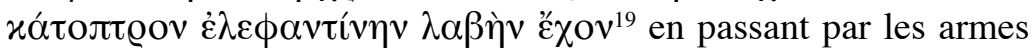

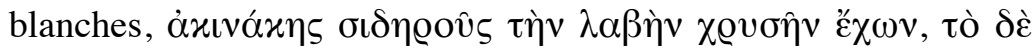

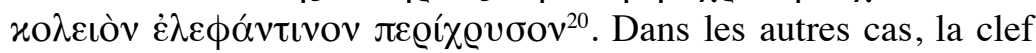

16. Dans un registre analogue, on note dès l'Indépendance des tuiles provenant du toit d'un temple et rangées dans une boîte en papyrus, témoignant ainsi de leur valeur votive, ID $443 \mathrm{Bb}$ (138): elles ont pu avoir une réelle utilité mais n'en restent pas moins enregistrées parmi les offrandes.

17. Sur l'inventivité lexicale des inventaires déliens, Cl. Prêtre, «Erreurs de gravure volontaires et involontaires dans les inventaires déliens: de la création d'hapax à l'usurpation d'identité», Tekmèria 8 (2003/2004), p. 86-101.

18. $I G \mathrm{XI} 2,287 \mathrm{~B}(72)$ à

19. $I G \mathrm{II}^{2}, 1514$, II (23).

20. $I G \mathrm{II}^{2}, 1421$, II (27) 
est toute en métal, du fer quand il est indiqué, et ne présente aucune caractéristique. L'usage est évident dans trois occurrences sur six, puisque les rédacteurs de l'inventaire ont catalogué la clef dans le cadre d'une description des éléments de portes (on a également les bosselages et les clous décoratifs cités contextuellement). Pour les deux exemplaires suivants, il est plus malaisé d'en comprendre la destination exacte ni même la forme, en raison du caractère lacunaire et laconique des enregistrements. Relevons qu'il s'agit là des deux modèles les moins décrits, et on peut imaginer qu'ils appartiennent donc plus à un petit mobilier votif, comme un coffre par exemple, qu'à des portes de sanctuaire qui auraient sans doute été évoquées dans l'inventaire. La dernière clef mentionnée présente en revanche une vocation dédicatoire évidente: une sousprêtresse a fait l'offrande de cette clef de cleidouque lorsqu'elle a reçu sa charge de prêtrise. L'ornementation sur l'objet, avec le lion protecteur, ainsi que sa destination en font une dédicace de valeur plus qu'un don utilitaire.

Ce passage en revue des clefs enregistrées dans les catalogues d'offrandes déliennes peut à présent être étoffé par l'étude des correspondants archéologiques de ces realia dans le sanctuaire d'Artémis à Thasos. Cinq exemplaires ont été retrouvés au cours des fouilles. Nous en donnons une description ci-dessous :

- Inv. 75.028. Clef en bronze, avec anneau de préhension large et plat et panneton à 10 dents (3/4/3). (fig. 1 droite)

- Inv. 76.011. Clef en bronze, avec anneau de préhension fin et panneton à 2 dents corrodées.

- Inv. 80.227. Clef en bronze très corrodée, dont il ne reste que le départ de l'anneau et le panneton à 2 dents.

- Inv. 81.015. Clef en bronze, avec anneau de préhension de section ronde et panneton à 2 dents.

- Inv. 81.057. Clef en bronze, avec anneau de préhension large et plat et panneton à 8 dents dont une cassée. (fig. 2 gauche)

Leur datation s'échelonne entre la fin du $\mathrm{v}^{\mathrm{e}}$ et le $\mathrm{III}^{\mathrm{e}} \mathrm{s}$. av. J.-C. d'après les données stratigraphiques en notre possession.

Sans reprendre ici l'histoire de la clef dans la Grèce antique, il est cependant nécessaire d'en livrer rapidement les deux grands types. 

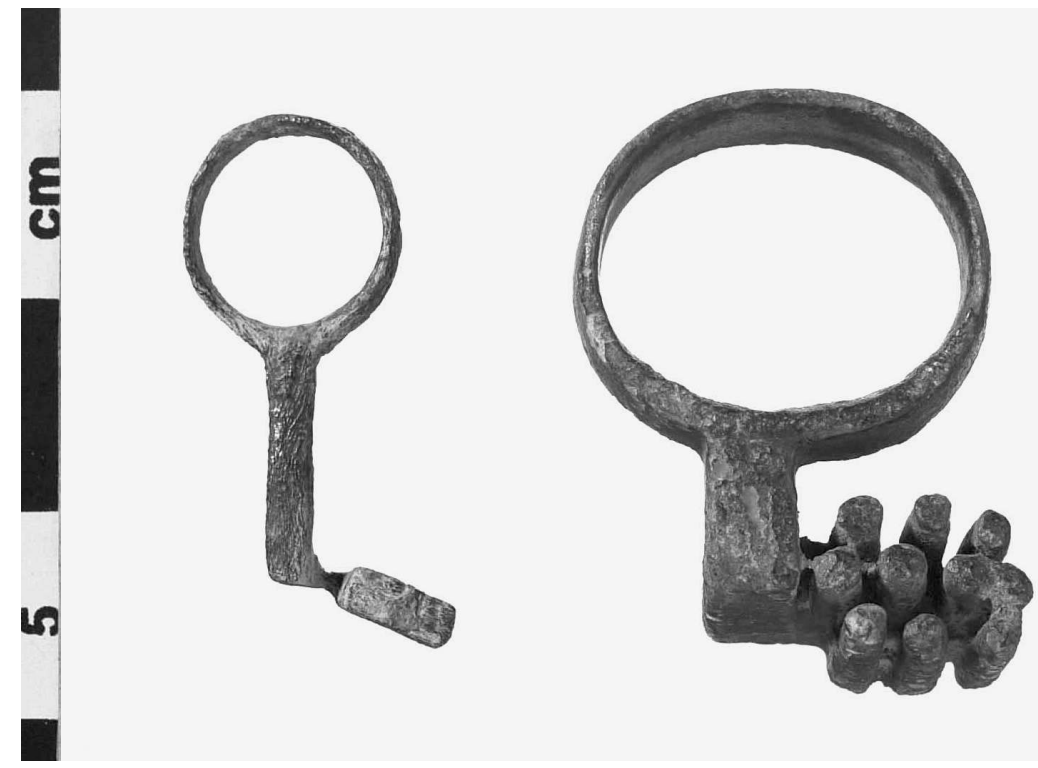

Fig. 1. Clefs en bronze, Artémision de Thasos. Cliché EFA, Ph. Collet.
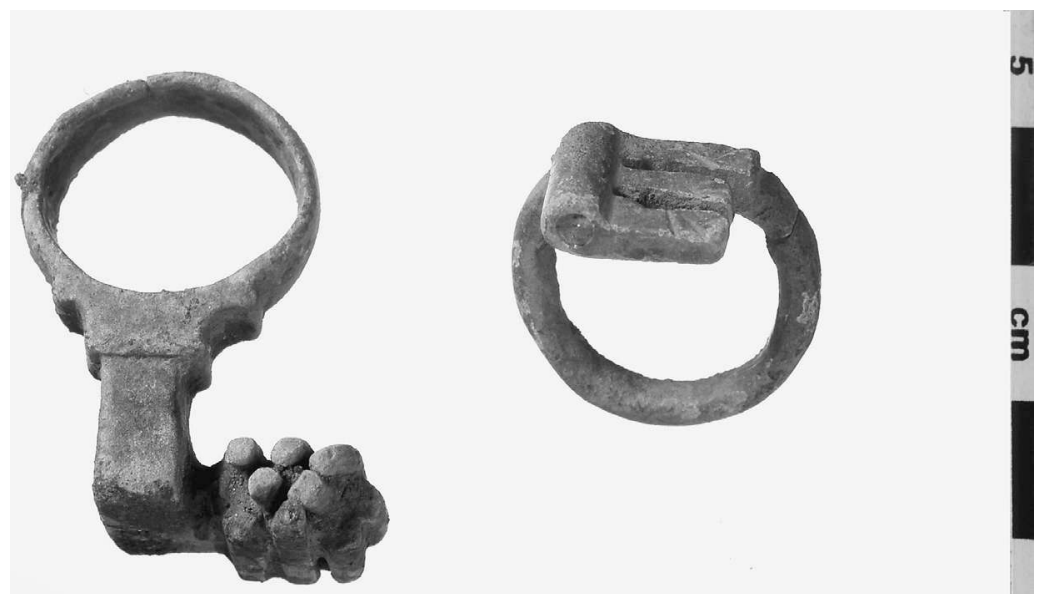

Fig. 2. Clefs en bronze, Artémision de Thasos. Cliché EFA, Ph. Collet. 
Le premier est appelé «clef de temple» en raison de son usage presque uniquement religieux. La clef, de grande taille, consistait en une tige de métal coudée en deux endroits, à extrémité élargie d'un côté et souvent terminée par une boule de l'autre. Le système complexe permettait de pousser un verrou mobile situé à l'intérieur de la porte, qu'on dégageait grâce à cette clef. Connue dès Homère ${ }^{21}$, elle perdure dans la plupart des sanctuaires grecs même après l'apparition de la serrure dite «laconienne» qui exige alors une clef plus perfectionnée pour s'adapter au verrou à pitons mobiles.

$\mathrm{La}$ «clef laconienne» fait son apparition en Grèce au vie s. et aurait été importée d'Égypte. Elle doit son nom à son pseudo-inventeur, Théodoros de Samos qui l'aurait introduite à Sparte ${ }^{22}$. Son originalité tient à sa forme, courbée à angle droit, qui porte à l'origine trois dents sur son panneton. Le besoin de sécurité augmentant au fil des siècles, le nombre de dents de la clef augmente parallèlement ${ }^{23}$. Elle est indissociable du verrou à balanoi, composé d'un même nombre de trous dans lesquels s'enfonçaient les dents de la clef. Une fois la clef enclenchée, ses dents poussaient les petites chevilles mobiles et on pouvait ainsi actionner le verrou et libérer la porte. Le même système s'appliquait aussi à des fermetures de coffrets et de boîtes avec des clefs de plus petite taille ${ }^{24}$.

À Thasos, toutes les clefs retrouvées dans l'Artémision appartiennent à la deuxième catégorie et on a en outre mis au jour deux verrous perforés qui ne correspondent cependant pas aux clefs découvertes.

À Délos en revanche, l'association de plusieurs exemplaires aux portes des sanctuaires, tout comme l'offrande de la prêtresse en font à coup sûr des clefs de temple.

La correspondance entre les realia des inventaires déliens et les objets des fouilles thasiennes est donc impossible à établir. L'étude conjointe des deux champs est cependant fondamentale car elle permet d'avoir une idée complète des grandes catégories de clefs de la Grèce antique. Sans le témoignage épigraphique et sans les

21. Odyssée, I (442)

22. Pline, $H N$, VII 198 avec le terme clauis dentala.

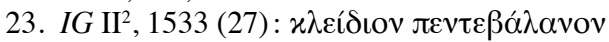

24. Pour un dessin et une explication du fonctionnement de ces clefs, Viktor Lilcic, «Une contribution à l'étude de la clé antique», Macedonian Acta Archaeologica 13 (1992), p. 201-212. 
données archéologiques, il n'aurait pas été possible de se représenter la variété typologique.

La seconde offrande délienne que nous tenterons de mettre en rapport avec les fouilles thasiennes appartient au domaine féminin de la parure et constitue une énigme comme il en existe de nombreuses

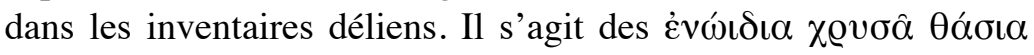
mentionnés au singulier dans un autre texte sous la forme $\dot{\varepsilon} v \omega ́ \tau \tau$ เov

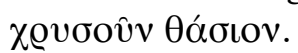

Le terme $\dot{\varepsilon} v \omega ́ \tau \iota$ v provient d'une hypostase de « $\dot{\varepsilon} v \omega \dot{\omega} \tau i$ », «dans l'oreille», et désigne ce qu'on nomme improprement «boucle

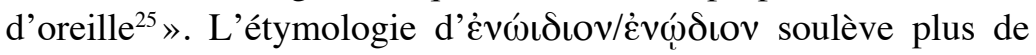
problèmes: pour $\mathrm{P}$. Chantraine ${ }^{26}$, deux solutions s'offrent selon que

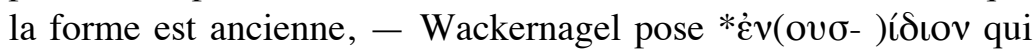

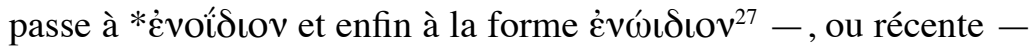
la forme supposerait une syncope d'origine familière à partir du diminutif $\dot{\varepsilon} v \omega \tau i ́ \delta ı v$. La synonymie entre les deux termes est de toute façon assurée. La forme attique évó

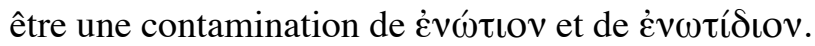

Si l'usage de ce terme est attesté depuis l'époque classique, c'est à l'époque hellénistique qu'on le mentionne davantage dans les sources littéraires et épigraphiques. Son emploi coïncide avec la

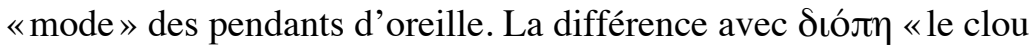
d'oreille percée» se marque de façon terminologique et formelle. «Dans l'oreille» ne signifie pas forcément «à travers», comme

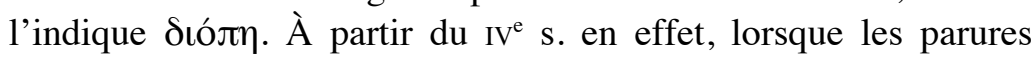
d'oreille s'alourdissent sous le poids des pendeloques, on utilise un nouveau système d'attache, destiné à ne pas déchirer le lobe. Il ne s'agit plus de passer à travers l'oreille par un trou percé à cet effet, mais de pincer le lobe en le pliant, de manière à retenir le bijou

25. Sur la nomenclature des types de bijoux et notamment la différence entre pendant d'oreille et boucle d'oreille, Gérard Nicolini, Techniques des ors antiques : la bijouterie ibérique du VII au IV $s$. (1990), volume I, première partie.

26. P. Chantraine, DELG s.v. oûs. «La forme attique غ̇vódıov semble être une contamination de $\dot{\varepsilon} v \omega ́ \tau \imath$ v et de $\dot{\varepsilon} v \omega \tau i ́ \delta เ o v »$, P. Chantraine, La formation des

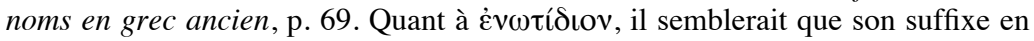

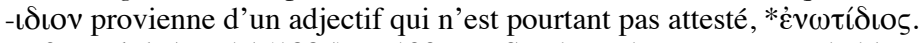

27. Phil. Anz. 15 (1885), p. 199 sqq. Son hypothèse est peu probable. Il est plus

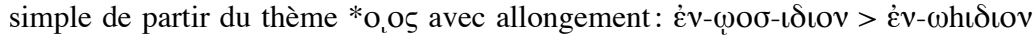
> Ėvódıov. 
d'oreille ${ }^{28}$. Ce type de suspension est sans doute lui aussi désigné par غ̇vótıov.

On peut donc imaginer que ce mot est utilisé pour distinguer clairement les lourds ornements qui pincent les oreilles par opposition aux clous d'oreilles simples (бı́́лๆ) ou par opposition aussi à l'anneau léger connu depuis plus longtemps et qui est mentionné

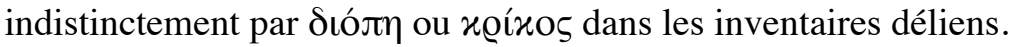

L'autre sens attesté d'èvótıv désigne, par élargissement, une pendeloque. Si l'on postule en effet qu'il s'agit d'un ornement d'oreille pendant, il est possible, par un effet de synecdoque, que l'ensemble, le pendant d'oreille, ait servi à nommer un autre élément, la pendeloque.

Les mentions de pendants d'oreilles sont nombreuses et variées dans les inventaires. Cependant, sous des formules différentes se cache bien souvent le même objet. À Délos, la forme Ėvótıov ne se rencontre que dans les inventaires de l'Indépendance tandis qu'èvóıtorov est le terme déjà employé au $v^{\mathrm{e}} \mathrm{s} .^{29}$. Il est conservé ensuite puisque les hiéropes utilisent indistinctement les deux graphies dans des inventaires chronologiquement rapprochés. Ainsi, en 199B (66)[274 av. J-C.], l'Eileithyaion possède un évóı

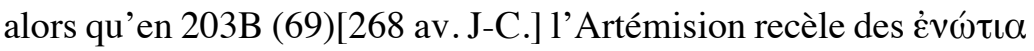

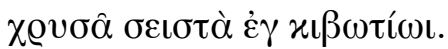

Sont résumées ci-dessous les différentes occurrences de ce type de bijou, et on notera que les offrandes sont essentiellement faites à des divinités féminines.

\section{Inventaires de l'Indépendance:}

Artémision:

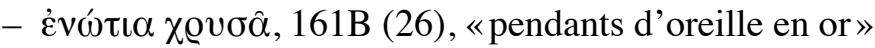

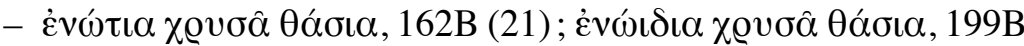
(46), «pendants d'oreille en or thasiens»

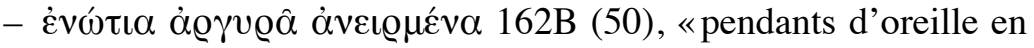
argent, non enfilés »

28. Cf. Dyfri Williams et Jack Ogden, Greek gold (1994), no 12 p. 60 où ce système est très visible: au vu du diamètre de la tige des pendants, il est en effet évident qu'elle ne passait pas à travers un trou dans l'oreille.

29. En ID 103 (25); 104 (51); 104 (83); 104-2B (4) etc. 


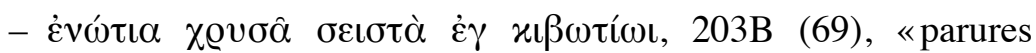
d'oreille en or pendantes dans une boîte»

Eileithyaion:

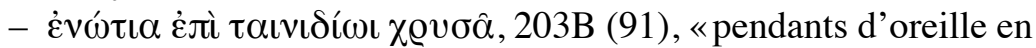
or sur une bandelette»

Temple d'Apollon:

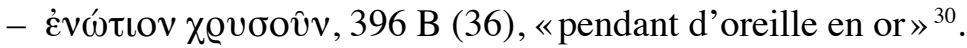

\section{Inventaires de la seconde domination athénienne:}

Aphrodision :

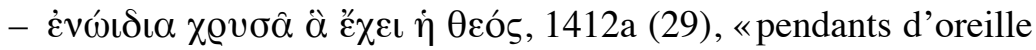
que porte la déesse »

Artémision en l'Île :

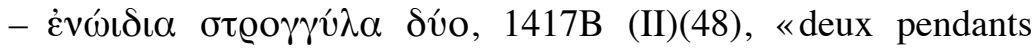
d'oreille ronds »

Thesmophorion:

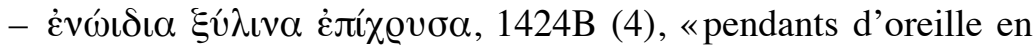
bois plaqué d'or ${ }^{31}$

Anoubideion:

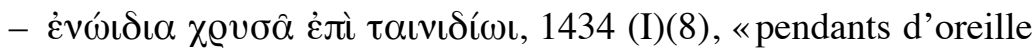
en or sur une bandelette»

Isideion :

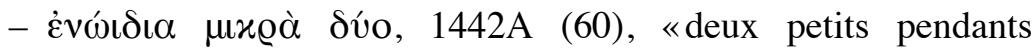
d'oreille»

Les exemplaires les plus intéressants de cette liste sont également les plus détaillés ${ }^{32}$. Grâce à la description des hiéropes, غ̇vótıov

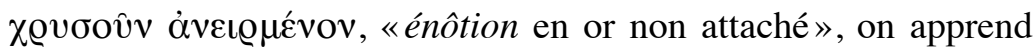

30. Il est étonnant de constater qu'aucun de ces bijoux ne se retrouve dans les inventaires de la seconde domination athénienne, qui en recèlent pourtant beaucoup d'autres.

31. Appartenant à une statue de la déesse, d'où la matière.

32. Les inventaires enregistrent encore beaucoup d'autres pendants d'oreille, mais sans précision aucune, ou dans le meilleur des cas, avec la mention de la matière. En produire la liste ici serait inutile et fastidieux. Tout au plus peut-on

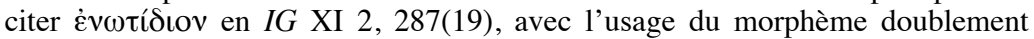
suffixé -ıঠıv. 
ainsi que les bijoux d'oreilles étaient composés de plusieurs éléments travaillés séparément: l'assemblage se faisait avec des

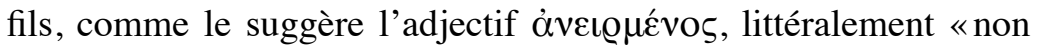

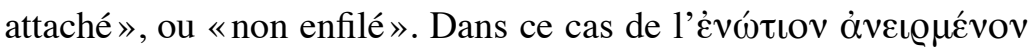
de l'Artémision, il est possible de traduire le mot par «pendeloque»: la mention d'un élément détaché n'est pas étonnante: les hiéropes enregistraient de façon scrupuleuse tous les fragments de bijoux. Cela confirme également l'idée que des pendeloques étaient peutêtre attachées à la partie fixe de la parure d'oreille et cela explique

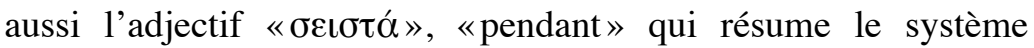
entier: un enôtion simple pinçant le lobe auquel sont accrochées des pendeloques.

Quelques autres modèles sont marquants. Ils permettent d'abord de faire la distinction entre les bijoux réels qu' on offrait pour décorer la statue de la déesse et les faux bijoux intégrés à son ornementation, de matière non durable, tel le bois doré à la feuille.

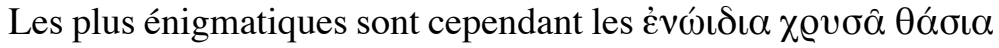
«pendants d'oreille thasiens». C'est à l'Artémision délien qu'on note leur dédicace. Leur poids, 5 drachmes ${ }^{33}$, est stable et très léger, si on compare à d'autres offrandes déliennes (à titre d'exemple, une couronne fait 100 drachmes, un anneau 2 drachmes, le fameux collier d'Ériphyle 156 drachmes).

L'origine géographique de cette offrande n'aurait en soi rien d'extraordinaire. Par ailleurs, on connaît par les sources littéraires tardives plusieurs attestations de productions dites «thasiennes».

Elles se répartissent en trois catégories :

- les fruits secs

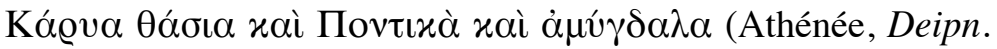

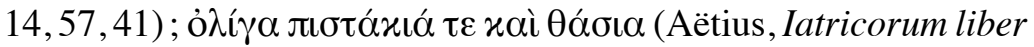

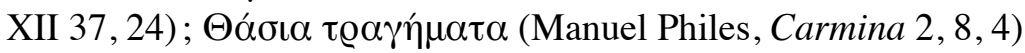

- les amphores et autres stamnoi

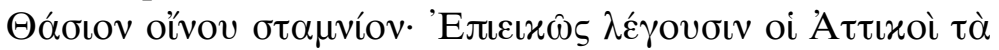

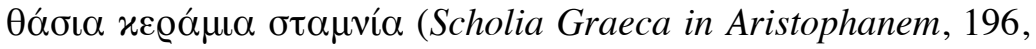

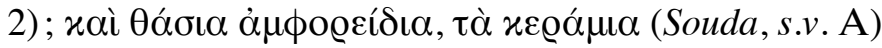

- la saumure et la grasse marinade

33. Hormis une erreur due au lapicide dans une des trois mentions. Cinq drachmes correspondent en gros à 21 grammes. 


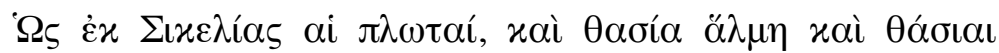

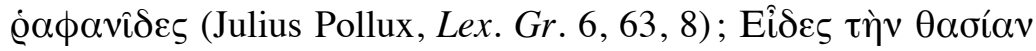

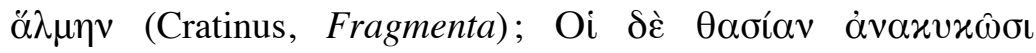

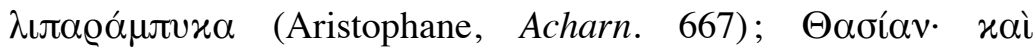

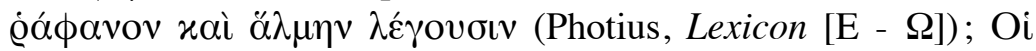

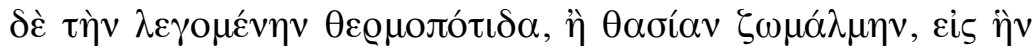

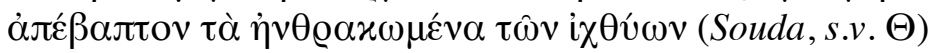

On remarquera cependant qu'il n'y a jamais de référence à un bijou de facture thasienne. L'identification de ce type de parure d'oreille est donc problématique.

L'usage desépithètes géographiquesest mal systématiséet souvent

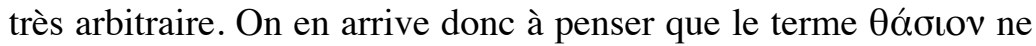
souligne pas forcément la particularité ethnique de ce bijou. $\mathrm{Ph}$. Bruneau avait travaillé sur le problème des épithètes géographiques en recherchant d'abord les trois raisons d'une telle nomenclature: était-ce une indication de l'origine réelle d'un objet, une information sur l'origine du type, ou épithète purement descriptive? Au terme de sa démonstration, il admettait que l'épithète géographique est «souvent moins le reflet de l'origine historique des produits ainsi désignés qu'un mode de dénomination typologique ${ }^{34} »$. Dès l'Antiquité, il n'était donc pas plus sûr de pouvoir distinguer $e x$ abrupto des pendants «thasiens» que des étoffes amorginiennes. Il avait dû y avoir forcément à l'origine un type de pendant d'oreille qui avait été fabriqué à Thasos et qui était suffisamment original pour qu'on le nomme ainsi, mais ensuite, les liaisons commerciales aidant, rien n'empêchait qu'un artisan de Syros, du Péloponnèse ou d'Eubée reproduise ce modèle en gardant la terminologie première mais sans plus forcément la comprendre.

Les mentions d'ėvóıঠı chez les auteurs littéraires et dans les autres inscriptions sont nombreuses. Dans les inventaires attiques par exemple, on relève différents types de bijoux d'oreille, dédiés

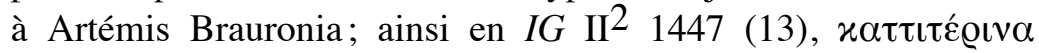

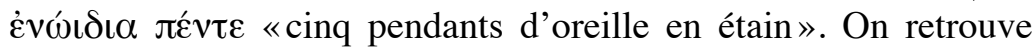
également d'autres bijoux semblables dans la parure de la Niké de

34. Philippe Bruneau, «D'un Lacedaemonius orbis à l'aes Deliacum», Mélanges Plassart (1976), p. 15-45 et particulièrement p. 36 sqq. pour un dépouillement des épithètes géographiques. 
l'Hékatompédon. D. Burr Thompson les rassemble dans un article où elle donne sa propre définition du terme évóıঠıov lorsqu'il est fait référence à cette parure: "The etymology of this word is selfevident. It is used by Aischylos (Frg 102) and elsewhere on the treasure-lists. Types of earrings that were popular in the late fifth century were the disk leech and simple pendant, which the rosette disk with daugling inverted pyramid appears on the Parthenon as shown by the Aspasios gem and the Kul Oba medaillions (Olynthus, IV, $\mathrm{n}^{\circ} 410$ ). In the fourth century, a more elaborate type possibly with a pendant of a flying Nike would be probable ${ }^{35} »$. Sa description, qui s'applique uniquement aux inventaires attiques, rejoint pourtant la nôtre, pour décrire les bijoux déliens. Seul le pendentif en forme de Niké moins courant dans les inscriptions déliennes, est souvent remplacé par d'autres pendeloques, comme nous le verrons ensuite.

Le mot apparaît également en 250 av.J.-C.à Tanagra par exemple, dans le catalogue d'offrandes présentées par les femmes préposées à la garde-robe sacrée des déesses Déméter et Perséphone ${ }^{36}$.

Dans la littérature, la description la plus complète est celle d'Énée le Tacticien au IV s., lorsqu'il décrit un stratagème utilisé

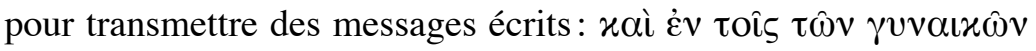

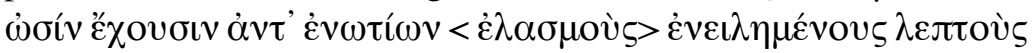
$\mu \mathrm{o} \iota \beta \delta$ ívov, , et ils placent aux oreilles des femmes des lamelles de plomb [écrites] et roulées sur elles-mêmes, en guise de parures d'oreille.» $(31,7)$ On doit supposer des modèles confectionnés à partir de ces minces rouleaux de métal repliés sur eux-mêmes, à moins qu'il s'agisse de pendeloques suspendues à une tige quelconque.

Ce passage en revue des occurrences textuelles donne un bon aperçu de la fréquence des èvóı $\delta\llcorner\alpha$ aux époques classique et hellénistique, mais il n'y a aucune allusion à une facture thasienne réputée pour ce genre de bijou.

L'examen des sources épigraphiques et littéraires dans ce cas, doit alors être complété par l'étude des objets trouvés lors des fouilles thasiennes, qu'ils soient en situation votive ou dans des habitations,

35. Dorothy Burr Thompson, «The golden Nikai reconsidered», Hesperia 13 (1944), 173-209.

36. Théodore Reinach, «Un temple élevé par les femmes de Tanagra», REG 12 (1899), p. 71. 
voire des tombes. Ainsi en 2004, il m'avait été permis d'examiner les bijoux provenant des tombes du port alors fouillé3 ${ }^{37}$. Outre les couronnes, les bagues, et les bracelets de petite taille, quelques boucles d'oreille avaient été mises au jour sans qu'il soit possible de trouver un correspondant fiable à la description délienne. En effet, les rares types alors découverts ne présentaient aucune particularité qui les désignât comme thasiens ${ }^{38}$.

Y. Grandjean avait trouvé un exemplaire de boucle d'oreille en bronze près de la porte du Silène, qu'il décrit ainsi: «boucle d'oreille en bronze, spiraliforme, se terminant aux deux extrémités par deux renflements successifs ${ }^{39}{ }^{»}$.

À l'Artémision enfin, seuls deux exemplaires d'énôtion, de facture identique à celle du Silène, ont pu être recensés. L'un d'eux est en bronze et ne comporte ni incision ni ornementation, tandis que l'autre présente l'intérêt d'être en argent, avec à chaque extrémité, un motif en relief sculpté avec incisions et pyramide terminée à son sommet par une boule. Dans ces trois cas, il ne s'agit cependant pas d'une facture propre à Thasos, puisque on a des bijoux semblables dans tous les grands sanctuaires du monde grec ${ }^{40}$.

C'est là la singularité de cette étude: en dépit des mentions épigraphiques d'un type de bijou thasien, et en dépit aussi des centaines d'objets votifs recensés dans le matériel archéologique de Thasos, le nombre des bijoux d'oreille retrouvé est très infime ${ }^{41}$ : très infime par rapport au nombre total d'objets, très infime par rapport à ce qu'on observe en général comme dédicaces dans des sanctuaires et surtout très infime par rapport à ce qu'on aurait pu espérer en

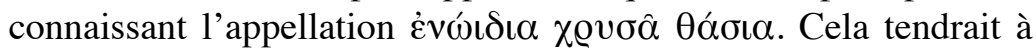

37. Essentiellement des tombes du IV $\mathrm{e}$.

38. Dans le matériel auquel j'avais eu accès. La poursuite des fouilles a peutêtre permis d'infirmer cette assertion mais en l'absence de renseignements précis sur les types de boucles d'oreille découverts ensuite, il est préférable de ne pas argumenter davantage.

39. Y. Grandjean, Recherches sur l'habitat thasien à l'époque grecque, T. 1, p. $253, \mathrm{n}^{\circ} 16$.

40. David M. Robinson, Excavations at Olynthus, X (1941), p. 89-91, et pl. 18, $n^{\circ} 312-313$. Dernièrement une série analogue, encore inédite, a été trouvée à Kythnos par Alexandros Mazerakis-Ainian.

41. Autre paradoxe qui consolera de ce maigre butin: à Délos, on n'a pas davantage trouvé d'enôtia dans les fouilles, alors que leur fréquente dédicace n'est plus à démontrer. 
confirmer l'hypothèse avancée ci-dessus: le bijou n'aurait plus de thasien que le nom.

On termine donc cette recherche par un constat d'aporie contradictoire car on se heurte à un paradoxe souvent dû aux aléas des fouilles; les inscriptions et les textes fournissent beaucoup de renseignements sur les offrandes à une divinité et la réalité archéologique paraît parfois bien mince en comparaison. Cela explique donc pourquoi il est difficile de comprendre, dans l'état actuel de nos connaissances, ce que des èvótı $\theta \alpha ́ \sigma ı \alpha$ pouvaient avoir comme caractéristique qui dépassât les frontières insulaires. De même, si les grands types de clefs mentionnés dans les sanctuaires sont bien déterminés, on ne trouve pas forcément leurs correspondants dans les objets issus des fouilles.

La démarche entreprise ci-dessus, en dépit de ses résultats nuancés, devrait cependant pouvoir démontrer l'intérêt et le riche potentiel qu'il y a à ne pas se cantonner à l'étude du matériel votif stricto sensu. En le replaçant dans un contexte littéraire et épigraphique, on peut obtenir des analyses plus fines et comprendre bien des modes de fonctionnements votifs.

clarisse.pretre@gmail.com 